\section{Naturlatexallergie bei Spina bifida - ein Sonderfall}

Patienten, die mit einer Spina bifida geboren wurden, zeigen auffällig häufig Allergien gegen Naturlatex. Ist dies allein eine Folge der zahlreichen Operationen, denen sich die Betroffenen unterziehen müssen, oder kommen zusätzliche Faktoren ins Spiel?

ür die Sensibilisierung gegen Naturlatex gelten direkter Hautkontakt, Inhalation - bei Verwendung gepuderter Naturlatexhandschuhe - oder Schleimhautkontakt als Voraussetzung. Spinabifida-Patienten haben extrem häufig Naturlatexallergien. Die Prävalenz liegt je nach Untersuchung zwischen 30\% und $100 \%$.

Ein Team aus Pädiatern und Dermatologen der Universität Wien überprüfte das Vorliegen einer Sensibilisierung gegen Naturlatex bei 35 Kindern mit Spina bifida. Die so ermittelte Prävalenz verglichen sie mit derjenigen bei
20 Kindern mit Gastroschisis oder Omphalozele sowie bei 45 Kindern mit posthämorrhagischem Hydrozephalus. Die Patienten mit Hydrozephalus waren vier bis sechs Wochen nach der Geburt operiert worden, alle anderen bereits in den ersten Lebenstagen. Die primäre Behandlung hatte nicht unter Naturlatexfreien Bedingungen stattgefunden.

Bei 46\% der Kinder mit Spina bifida fand sich spezifisches IgE gegen $\mathrm{Na}$ turlatex. Dies war signifikant häufiger als bei den Kindern mit posthämorrhagischem Hydrozephalus (8,9\%) und Kindern mit Gastroschisis oder Om- phalozele (5\%). Auch eine Subgruppenanalyse bei Patienten mit höchstens drei Operationen und vergleichbarer $\mathrm{Zu}$ sammensetzung bezüglich Geschlecht und Alter zeigte eine signifikant häufigere Sensibilisierung bei den Spina-bifida-Patienten. Die Autoren vermuten, dass bei diesen Patienten zusätzliche Faktoren wie spezifische HLA-Typen für die größere Häufigkeit von Naturlatexallergien verantwortlich sind.

Fazit: Kinder mit Spina bifida weisen im Vergleich zu Kindern, die wegen anderer angeborener oder perinatal erworbener Fehlbildungen ebenfalls in den ersten Lebenswochen mehrfach operiert wurden, signifikant häufiger eine Sensibilisierung gegen Naturlatex auf.

Eiwegger T et al. Early exposure to latex products mediates latex sensitization in spina bifida but not in other diseases with comparable latex exposure rates. Clin Exp Allergy 2006; 36: 1242-46

\title{
Anti-IgE bei schwerer Kälteurtikaria
}

\section{Der Anti-IgE-Antikörper Omalizumab ist seit 2005 zur Behandlung von schwerem allergischen Asthma bronchiale zugelassen. Theoretisch sollte die IgE-Blockade auch bei anderen allergischen Erkrankungen eine Besserung erbringen - eine davon könnte Kälteurtikaria sein, wie ein Fallbericht aus den USA zeigt.}

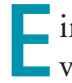
12-Jährige hatte, nachdem sie von Florida in den Nordosten der USA umgezogen war, bei kaltem Wetter wiederholt juckende Hautausschläge entwickelt. Dabei reagierte sie zunächst vor allem auf kalte Flächen und kaltes Wasser. Die Symptome verschwanden 30-60 Minuten nach Gabe von Diphenhydramin. Innerhalb von zwei Jahren nahmen Häufigkeit und Schwere der Schübe zu, wobei auch systemische Beschwerden auftraten. Zudem reagierte das Mädchen bereits auf kalte Luft oder Nahrungsmittel. Da die atopische Patientin darüber hinaus auch an Asthma litt, bestand ihre Vortherapie aus inhalativen Glukokortikoiden und $\beta_{2}$-Agonisten sowie Leukotrienrezeptorantagonisten und Antihistaminika.
Basierend auf der klinischen Anamnese, dem Fehlen von systemischen Erkrankungen und Kryoproteinen sowie durch positiven Eiswürfeltest wurde eine idiopathische Kälteurtikaria diagnostiziert. Da diese progredient verlief, wurde ein Therapieversuch mit Omalizumab in einer Dosis von $375 \mathrm{mg}$ alle zwei Wochen unternommen. Nach der zweiten Injektion reagierte die 12-Jährige auf kalte Getränke nur noch mit mildem Pruritus. Nach etwa zwei Monaten traten bei Kontakt mit kalter Luft und kaltem Wasser nur noch milde Symptome auf - nach fünf Monaten waren auch diese verschwunden. Der Eiswürfeltest wurde negativ und Begleittherapien konnten reduziert oder abgesetzt werden.

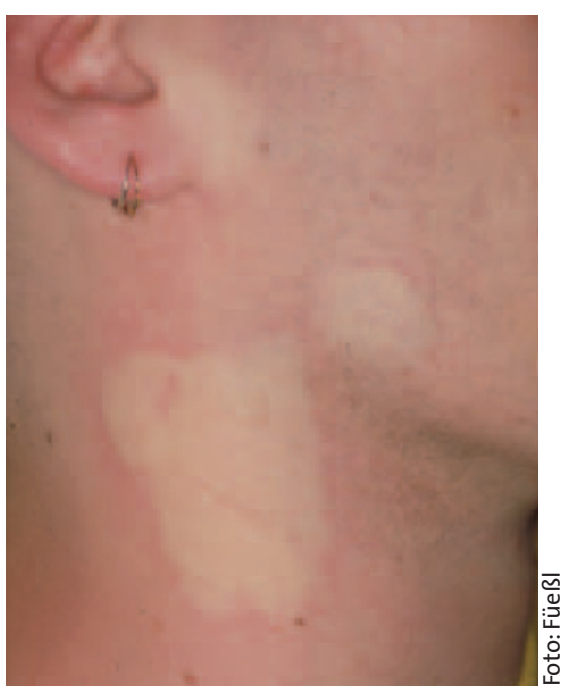

\section{Kälteurtikaria durch Eispackung}

Fazit: Dieser Fall zeigt erstmals, dass ein Patient mit schwerer Kälteurtikaria von der Gabe eines Anti-IgE-Antikörpers profitieren kann. Er bestätigt zudem die Bedeutung von IgE in der Pathogenese der idiopathischen Kälteurtikaria. pe

Boyce JA. Successful treatment of cold-induced urticaria/anaphylaxis with anti-lgE. J Allergy Clin Immunol 2006; 117: 1415-8 